\title{
Bruton's tyrosine kinase inhibition effectively protects against human IgE-mediated anaphylaxis
}

\author{
Melanie C. Dispenza, ${ }^{1,2}$ Rebecca A. Krier-Burris, ${ }^{2}$ Krishan D. Chhiba, ${ }^{2}$ Bradley J. Undem, ${ }^{1}$ Piper A. Robida, ${ }^{2}$ and Bruce S. Bochner ${ }^{2}$ \\ 'Division of Allergy and Clinical Immunology, Department of Medicine, Johns Hopkins University School of Medicine, Baltimore, Maryland, USA. ²Division of Allergy and Immunology, Department of Medicine, \\ Northwestern University Feinberg School of Medicine, Chicago, Illinois, USA.
}

\begin{abstract}
No known therapies can prevent anaphylaxis. Bruton's tyrosine kinase (BTK) is an enzyme thought to be essential for highaffinity IgE receptor (Fc\&RI) signaling in human cells. We tested the hypothesis that FDA-approved BTK inhibitors (BTKis) would prevent IgE-mediated responses including anaphylaxis. We showed that irreversible BTKis broadly prevented IgEmediated degranulation and cytokine production in primary human mast cells and blocked allergen-induced contraction of isolated human bronchi. To address their efficacy in vivo, we created and used what we believe to be a novel humanized mouse model of anaphylaxis that does not require marrow ablation or human tissue implantation. After a single intravenous injection of human CD34+ cells, NSG-SGM3 mice supported the population of mature human tissue-resident mast cells and basophils. These mice showed excellent responses during passive systemic anaphylaxis using human IgE to selectively evoke human mast cell and basophil activation, and response severity was controllable by alteration of the amount of allergen used for challenge. Remarkably, pretreatment with just 2 oral doses of the BTKi acalabrutinib completely prevented moderate IgE-mediated anaphylaxis in these mice and also significantly protected against death during severe anaphylaxis. Our data suggest that BTKis may be able to prevent anaphylaxis in humans by inhibiting Fc\&RI-mediated signaling.
\end{abstract}

\section{Introduction}

Anaphylaxis is an acute, potentially life-threatening systemic allergic reaction characterized by urticaria, angioedema, bronchospasm, nausea/vomiting, abdominal cramping, diarrhea, hypotension, and/or shock (1). IgE-mediated anaphylaxis requires antigen cross-linking of FceRI-bound IgE on the surface of mast cells and basophils, initiating the release of mediators including histamine, tryptase, prostaglandins, leukotrienes, and cytokines (2). No known therapies are capable of reliably preventing anaphylaxis, so standard of care entails avoidance of triggering agents as well as prompt treatment with epinephrine after a reaction has already begun (1). However, allergen avoidance is not always possible, and accidental exposures often occur, especially in the case of foods or stinging insects. In addition, patients are intentionally exposed to known allergens during diagnostic and therapeutic procedures such as allergen skin testing, immunotherapy, and drug or food desensitizations, all of which have a risk of a serious and/or life-threatening reaction (1, 3). Unfortunately, even with rapid and comprehensive medical treatment, anaphylaxis can be fatal (1). Therefore, there is an unmet need for therapies that can reduce the frequency and/or severity of IgE-mediated anaphylactic reactions from both accidental and therapeutic exposures to allergens.

Bruton's tyrosine kinase (BTK) is a key component of FceRI signaling in human mast cells and basophils (4-6), and thus rep-

Conflict of interest: The authors have declared that no conflict of interest exists. Copyright: (5) 2020, American Society for Clinical Investigation.

Submitted: April 2, 2020; Accepted: May 28, 2020; Published: August 4, 2020.

Reference information: J Clin Invest. 2020;130(9):4759-4770.

https://doi.org/10.1172/JCl138448 resents an attractive target for preventing IgE-mediated responses in these cells. Based on the dependence of B cell signaling and survival on BTK activity (7), pharmacologic agents targeting BTK are now FDA-approved for the treatment of $\mathrm{B}$ cell malignancies. Ibrutinib and acalabrutinib are both oral, FDA-approved irreversible BTK inhibitors (BTKis) and are generally well tolerated (8-10). We have previously shown in a small pilot study that 2 standard doses of ibrutinib can reduce or eliminate skin prick test reactivity to peanut and tree nuts in healthy allergic adults, without any observed toxicity or side effects even when given for up to 1 week (11). Therefore, we hypothesized that clinically relevant doses of BTKis would successfully prevent IgE-mediated anaphylaxis.

\section{Results}

BTKis prevent IgE-mediated degranulation and cytokine release in primary human mast cells and basophils. We and others have previously shown that in vitro pretreatment of human basophils with the first-generation BTKi ibrutinib or the second-generation BTKi acalabrutinib abolishes their IgE-mediated activation (6, 12-14), and that 2 FDA-approved doses of ibrutinib prevents IgE-mediated basophil activation ex vivo (11). Prior studies have also demonstrated ibrutinib's ability to inhibit IgE-mediated degranulation in canine neoplastic mast cells (15). We therefore sought to investigate the effects of BTKis on IgE-mediated activation of human mast cells. Primary human skin-derived mast cells (SDMCs) were isolated from discarded normal skin fragments as previously published (16). After 4 weeks in culture, SDMCs from each donor achieved $70 \%-90 \%$ purity as assessed by flow cytometry (Supplemental Figure 1; supplemental material available online with this article; https://doi.org/10.1172/JCI138448DS1). SDMCs showed excellent IgE-mediated degranulation responses; the average 
percent of total $\beta$-hexosaminidase release of SDMCs following passive sensitization with human biotinylated IgE and crosslinking with streptavidin was $40.7 \%$ over unstimulated cells (data not shown). Pretreatment of SDMCs for 15 minutes with irreversible inhibitors of BTK (ibrutinib, acalabrutinib, or tirabrutinib) immediately before IgE-mediated activation completely abolished their degranulation in a dose-dependent manner as measured by both percent of total $\beta$-hexosaminidase release $(P<$ 0.0001 by 2-way ANOVA; Figure $1 \mathrm{~A}$ ) and upregulation of the surface activation markers LAMP1 and CD203c as assessed by flow cytometry $(P<0.0001$ for both markers; Figure 1B). Inhibition of degranulation was achieved at clinically relevant concentrations with average $\mathrm{IC}_{50}$ 's of 40, 222, and $309 \mathrm{nM}$ for ibrutinib, acalabrutinib, and tirabrutinib, respectively.

We next investigated whether or not BTKis could prevent IgE-mediated de novo cytokine synthesis in human mast cells. Data showed that BTKis significantly prevented IgE-mediated cytokine secretion from SDMCs in a dose-dependent manner (Figure $1 \mathrm{C})$. At $1 \mu \mathrm{M}$, ibrutinib and acalabrutinib reduced the release of IL- 6 from $1.98 \pm 0.62 \mathrm{pg} / \mathrm{mL}$ in vehicle-treated cells to $0.36 \pm 0.12$ and $0.33 \pm 0.13 \mathrm{pg} / \mathrm{mL}$, respectively $(P<0.0001$ by 2 -way ANOVA), IL-8 from $18.28 \pm 11.42$ to $0.14 \pm 0.08$ and $0.45 \pm 0.29 \mathrm{pg} / \mathrm{mL}$ $(P=0.0027)$, IL-10 from $0.27 \pm 0.17$ to $0.02 \pm 0.01$ and $0.02 \pm 0.01$ $\mathrm{pg} / \mathrm{mL}(P=0.0100), \mathrm{MCP}-1$ from $17.61 \pm 5.90$ to $4.01 \pm 1.70$ and $4.19 \pm 1.21 \mathrm{pg} / \mathrm{mL}(P<0.0001)$, and GM-CSF from $103.59 \pm 70.04$ to $0.07 \pm 0.02$ and $0.20 \pm 0.15 \mathrm{pg} / \mathrm{mL}(P=0.0024)$, and trended toward suppression of TNF- $\alpha$ production from $47.08 \pm 44.47$ to $0.04 \pm 0.01$ and $0.09 \pm 0.06 \mathrm{pg} / \mathrm{mL}(P=0.0768)$.

Inhibitory effects on SDMCs gradually waned over several days after a single 15-minute treatment and subsequent washout of irreversible BTKis before IgE cross-linking in the absence of drug $(P<0.0001$; Figure 1D), suggesting recovery due to de novo synthesis of new BTK over this time. In line with prior data, pretreatment of human basophils for 15 minutes in vitro with tirabrutinib, another second-generation inhibitor, was equally as effective ( $\left.\mathrm{IC}_{50} 336 \mathrm{nM}\right)$, albeit less potent, as ibrutinib ( $\mathrm{IC}_{50} 40 \mathrm{nM}$ ) and acalabrutinib $\left(\mathrm{IC}_{50} 150 \mathrm{nM}\right)$ at preventing IgE-mediated activation as assessed by flow cytometric basophil activation testing (Figure 1E). Collectively, our data demonstrate that irreversible BTKis consistently prevent IgE-mediated activation of human mast cells and basophils in vitro.

BTKis prevent IgE-mediated bronchoconstriction in human lung tissue. We have previously shown that IgE-mediated bronchial contractions are virtually abolished by blockade of both $\mathrm{H} 1$ histamine and cysteinyl leukotriene receptors in vitro (17). However, even $1 \%$ or $2 \%$ of total histamine release from human bronchial mast cells is sufficient to cause the strong contraction of airway smooth muscle, and thus even a near-complete blockade of $\mathrm{H} 1$ receptors in vivo cannot prevent bronchoconstriction during anaphylaxis. Given that BTKis can completely prevent IgE-mediated degranulation of human mast cells and basophils in vitro, we next tested the hypothesis that they would also eliminate IgE-mediated bronchoconstriction by preventing activation of tissue-resident mast cells in the lung. Dissected human bronchi were pretreated with ibrutinib or vehicle for 30 minutes, and then anti-IgEinduced bronchoconstriction was assessed via increases in isomeric tension as previously described (17). Pretreatment with ibrutinib nearly abolished IgE-mediated bronchial contraction in a dose-dependent manner $(P<0.0001$ by 2 -way ANOVA; Figure $2 \mathrm{~A})$. At $100 \mathrm{nM}$ and $1 \mu \mathrm{M}$, ibrutinib virtually eliminated contraction to even the maximally effective concentration of $1 \mu \mathrm{M}$ antiIgE, suggesting complete or near-complete blockade of histamine and cysteinyl leukotriene release. Ibrutinib had no effect on the maximal contraction of bronchial tissue achieved by cholinergic agonists; maximal contraction evoked by carbamylcholine averaged $6.1 \pm 1.0 \mathrm{~g}$ and $6.3 \pm 1.2 \mathrm{~g}$ in control- and ibrutinib-treated tissues, respectively $(P=0.7232)$. As expected, up to $1 \mu \mathrm{M}$ ibrutinib had no effect on contraction induced by exogenously applied histamine, signifying that bronchial histamine responsiveness remained intact $(P=0.2952$; Figure $2 \mathrm{~B})$. The average $\mathrm{EC}_{50}$ 's for histamine were $6.35 \pm 0.07 \mu \mathrm{M}$ in vehicle-treated and $6.55 \pm 0.21$ $\mu \mathrm{M}$ in ibrutinib-treated bronchi, and the average maximal histamine-induced contraction was $85 \%$ in both vehicle- and ibrutinibtreated tissues. These data suggest that clinically relevant concentrations of BTKis can prevent IgE-mediated bronchospasm through the inhibition of mast cell mediator release.

NSG-SGM3 mice inoculated with human CD34 $4^{+}$cells can be used as a novel humanized mouse model of anaphylaxis without prior irradiation or surgical implantation of human tissue. We next sought to test the ability of BTKis to prevent IgE-mediated anaphylaxis in a preclinical model in vivo. Because of differences in its role in FceRI signaling between murine and human cells, BTK elimination is not sufficient to prevent FceRI-mediated signaling in murine cells or anaphylaxis in WT mice, as we and others have shown (5, 18-20). We therefore sought to use "humanized mice" that are engrafted with human leukocytes including human mast cells and basophils. NOD scid gamma (NSG) mice are highly immunodeficient, making them ideal for humanization. In the NSG-SGM3 strain, transgenes for human IL-3, GM-CSF, and SCF allow for the expansion and maturation of human hematopoietic cells (21-23). Previously published models of passive systemic anaphylaxis (PSA) in NSG-SGM3 mice entail irradiation or chemical ablation of the murine bone marrow, surgical implantation of human fetal liver and thymic tissue, and subsequent i.v. injection of human cord blood CD34 ${ }^{+}$stem cells (HSCs), creating "bone marrow, liver, thymus" (BLT) mice. BLT mice demonstrate good engraftment of human leukocytes and significant responses during PSA, including its regulation by human mast cell-targeting agents $(24,25)$.

We sought to create a simpler and less burdensome humanized mouse model for use in PSA experiments. We hypothesized that NSG-SGM3 mice could adequately support the growth and maturation of functional human myelogenous leukocytes including mast cells and basophils without human fetal tissue implantation. Additionally, given that human IgE cannot bind to murine FceRI receptors (26), we hypothesized that irradiation of the murine bone marrow would not be necessary for such a model to be used for induction of passive anaphylaxis using human IgE. Our results show that after a single i.v. injection of HSCs at 3-4 weeks of age, NSG-SGM3 mice demonstrated early engraftment of human leukocytes in as few as 4 weeks after HSC injection, and continued to populate over time, resulting in extensive engraftment by 16 weeks (Figure 3A). Both human CD $45^{+}\left(\mathrm{hCD} 45^{+}\right)$and murine $\mathrm{CD}^{4} 5^{+}\left(\mathrm{mCD} 45^{+}\right)$leukocyte subsets were detected in 
A

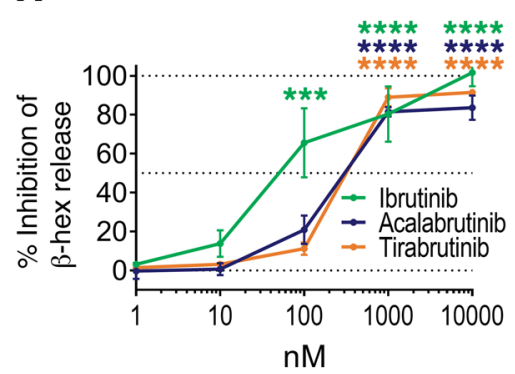

C

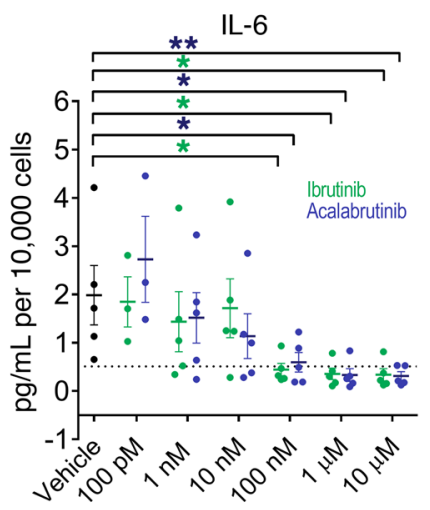

IL-8

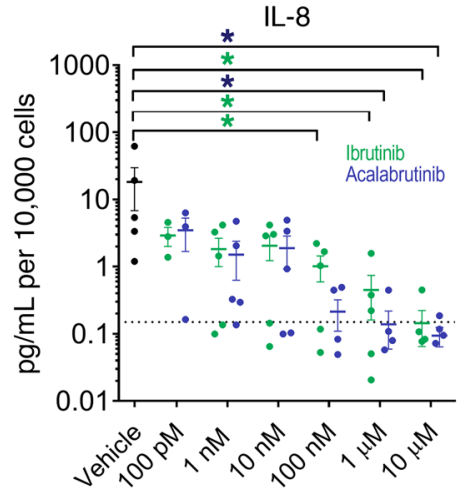

D

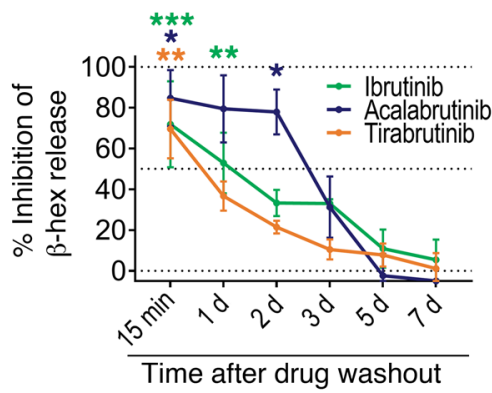

B

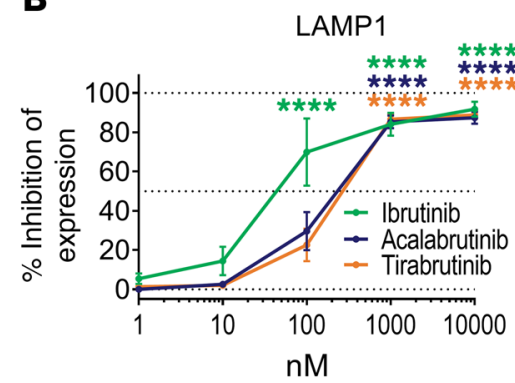

IL-10

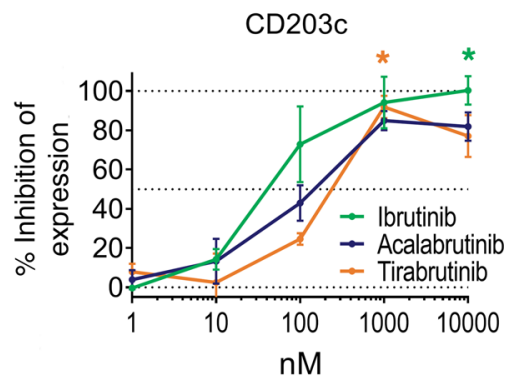

MCP-1

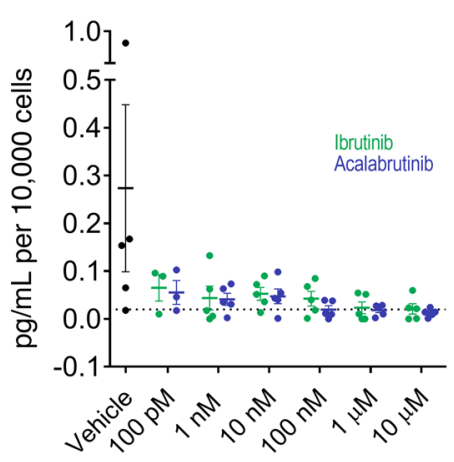

TNF- $\alpha$

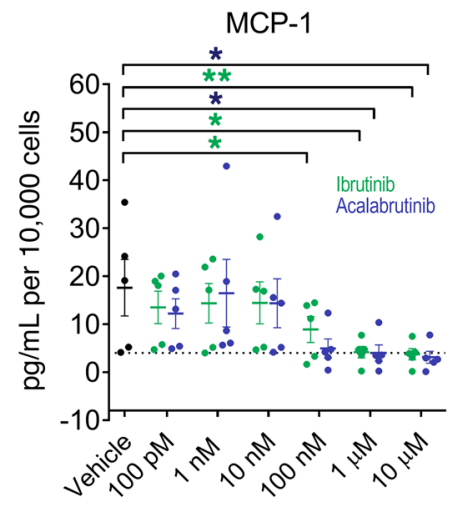

GM-CSF
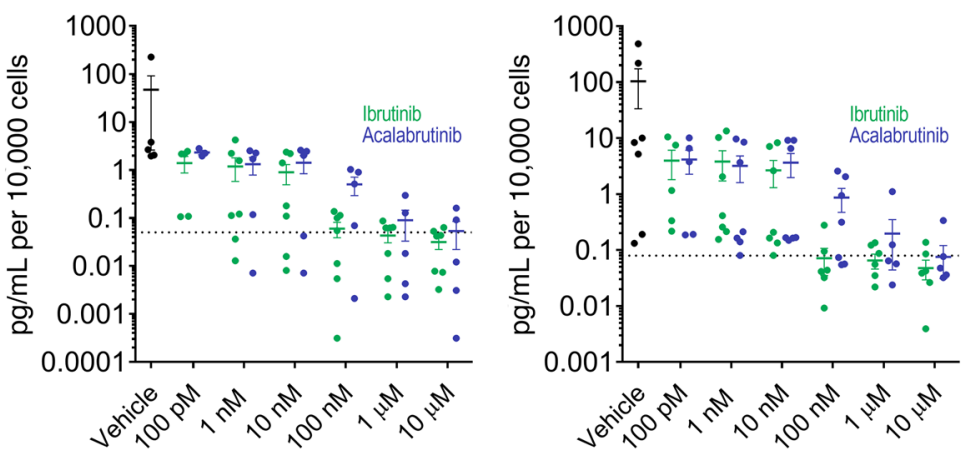

E

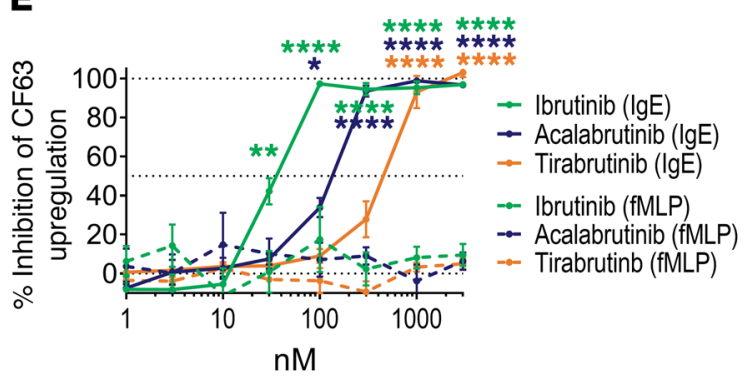

Figure 1. BTKis abrogate IgE-mediated mast cell and basophil activation and cytokine production in vitro. (A) Human SDMCs were passively sensitized with $50 \mathrm{ng} / \mathrm{mL}$ human biotinylated IgE overnight, then pretreated with BTKis for 15 minutes and activated for 1 hour with $100 \mathrm{ng} / \mathrm{mL}$ streptavidin to cross-link IgE. Percentage of total $\beta$-hexosaminidase ( $\beta$-hex) release was determined via colorimetric assay. $n=4-5$ using SDMCs from different donors. (B) SDMCs were treated with BTKis and activated as above, then incubated with fluorescently labeled antibodies against LAMP1 and CD203c before analysis by flow cytometry. Percentage of $\mathrm{LAMP}^{+}$and mean MFI of CD203c were measured in cKit ${ }^{+}$cells. $n=4$ different donors. (C) SDMCs were treated with BTKis for 15 minutes and then washed before IgE cross-linking as above. Twenty-four hours later, cytokine concentrations as indicated were assayed in supernatants using a fluorescent multiplex assay. $n=3-7$ different donors. Dotted lines indicate basal secretion by unstimulated cells. (D) To determine the duration of BTKis' effects, SDMCs were exposed to $1 \mu \mathrm{M}$ BTKis for 15 minutes and washed at the indicated time points before activation with IgE and assessment of $\beta$-hex release as above. $n=3$ different donors. (E) The indicated BTKis were added to anticoagulated human whole-blood samples for 15 minutes before activation with anti-FcERI $\alpha$ antibody (solid lines) or fMLP (dashed lines) as a control. Basophil activation was assessed by CD63 surface upregulation by flow cytometry. $n=4-6$ different donors. All data are displayed as means \pm SEM. ${ }^{*} P<0.05,{ }^{*} P<0.01,{ }^{* *} P<0.001,{ }^{* * * *} P<0.0001$ compared with vehicle-treated cells by 2-way ANOVA with repeated measures. 

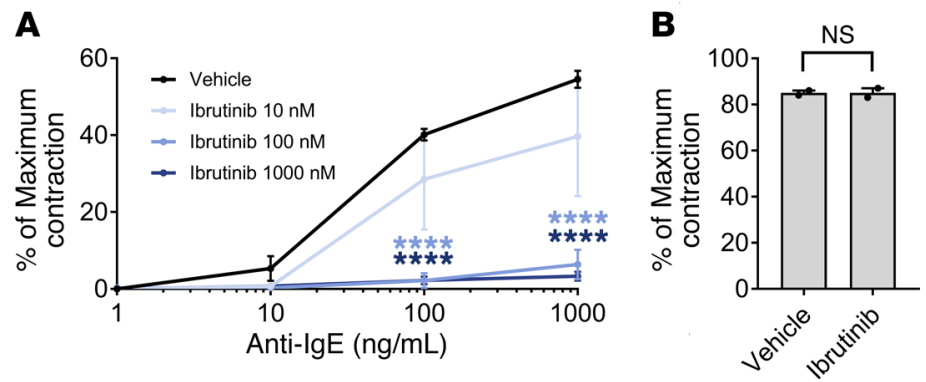

Figure 2. Ibrutinib effectively blocks anti-IgE-induced contraction of human bronchi. Isolated human bronchi were pretreated with ibrutinib or vehicle for 30 minutes, and then anti-IgE-induced contraction was measured. (A) Cumulative concentration-response curves for anti-IgE-induced contractions are shown for vehicle- and ibrutinib-treated tissues. Contraction is expressed as a percentage of the maximal obtainable contraction evoked by carbamylcholine $(100 \mu \mathrm{M})$ added at the end of the experiment. $n=4$ using bronchi from different donors. (B) Percentage of maximal contraction as achieved by treatment with exogenous histamine is shown for tissues treated with vehicle or $1 \mu \mathrm{M}$ ibrutinib. $n=2$ from different donors. All data are displayed as means \pm SEM. ${ }^{* * *} P<0.0001$ compared with vehicle-treated tissues by 2 -way ANOVA with repeated measures. NS, not significant by 2-tailed paired Student's $t$ test.

whole blood at all time points, though, as expected, hCD $45^{+}$cells increased over time, while $\mathrm{mCD} 45^{+}$cells proportionally decreased (Figure 3, B and H). Defined populations of hCD $45^{+}$Siglec- $8^{+}$ CD203 $\mathrm{c}^{+}$cells were observed in whole blood of engrafted mice, demonstrating the presence of circulating human mast cell and/ or basophil progenitors (Figure 3D). Populations of circulating human lymphocytes, eosinophils, monocytes, and NK cells were also observed (Figure 3, C, E, F, and G, respectively). At 16 weeks after HSC injections, mature human mast cells and basophils were detected in blood, spleen, bone marrow, and peritoneum as assessed by flow cytometry (Figure 4, A and C). Finally, mature human tissue-resident mast cells were detected in the organs of engrafted mice, including the skin, lung, intestine, stomach, and tongue (Figure 4, B, D, and E). Populations of mature murine mast cells were also observed, as expected (Figure 4, D and E). Interestingly, human mast cells constituted higher percentages of CD $45^{+}$ cells in the spleen (average 6.07\%), stomach (4.63\%), and peritoneal cavity $(41.77 \%)$ compared with murine mast cells $(0.15 \%$, $1.40 \%$, and $6.90 \%$, respectively), whereas human mast cells were less numerous in the lung (1.79\%), tongue (1.92\%), and skin (back skin $2.84 \%$, ear skin $0.61 \%$ ) compared with murine mast cells (1.98\%, 5.77\%, 9.12\%, and 7.25\%, respectively). Overall, these data demonstrate that NSG-SGM3 mice support the growth and maturation of human tissue-resident mast cells without prior marrow irradiation or surgical human tissue implantation.

NSG-SGM3 humanized mice serve as a robust model of human anaphylaxis wherein the response severity can be controlled. About 16 weeks after engraftment with a single i.v. injection of HSCs, NSG-SGM3 mice were passively sensitized using human chimeric IgE specific to 4-hydroxy-3-nitrophenylacetyl (NP). Mice showed a robust clinical response to subsequent NP-BSA challenge as demonstrated by a decrease in core body temperature and increase in clinical scoring (Figure 5, A and B, and ref. 27). In this model, alterations in the amount of NP-BSA antigen used for challenge produced differences in PSA response severity in a dose- dependent manner. For instance, $500 \mu \mathrm{g}$ of NP-BSA challenge resulted in severe and often fatal anaphylaxis (average maximum core temperature drop of $-4.47^{\circ} \mathrm{C}$, with $48 \%$ mortality), while challenge with a $20-\mu$ g dose of NP-BSA resulted in moderate anaphylaxis (average maximum temperature drop of $-3.91^{\circ} \mathrm{C}, 11 \%$ mortality), and administration of $5 \mu \mathrm{g}$ of NP-BSA resulted in mild anaphylaxis (average maximum temperature drop of $-3.11^{\circ} \mathrm{C}, 0 \%$ mortality). As expected, mice that were not engrafted with HSCs showed no response to human IgE sensitization during PSA, and mice sham-sensitized with PBS instead of IgE showed no anaphylactic response during challenge (Figure 5B). This preclinical mouse model involving human FceRI-bearing cells thus allowed for the investigation of BTKis' ability to prevent a range of PSA responses.

Acalabrutinib completely prevents moderate anaphylaxis during PSA in humanized mice. We next tested the hypothesis that BTKis can prevent anaphylaxis in vivo. Acalabrutinib had recently achieved FDA approval and was therefore chosen for use in mouse experiments for its higher selectivity for BTK and more favorable safety profile compared with ibrutinib. Engrafted NSG-SGM3 mice were sensitized with human anti-NP IgE as above and then pretreated with acalabrutinib by oral gavage 16 and 4 hours before NP-BSA challenge. Two doses of $15 \mathrm{mg} / \mathrm{kg}$ of acalabrutinib completely inhibited anaphylactic responses during moderate PSA (20 $\mu \mathrm{g}$ NP-BSA challenge) with an average maximum body temperature decline of $-0.369^{\circ} \mathrm{C}$ and $0 \%$ mortality compared with treatment with vehicle (average maximum temperature decline of $-3.678^{\circ} \mathrm{C}$ and $11 \%$ mortality; $P=0.0015$ by 2 -way ANOVA; Figure $6 \mathrm{~A}$ ). Mice receiving this dose of acalabrutinib also showed no increase in clinical score during PSA; consequently, based on scoring and core temperature, their PSA response was not significantly different from that of mice sham-sensitized with PBS instead of IgE. Two lower doses of $1.5 \mathrm{mg} / \mathrm{kg}$ of acalabrutinib provided partial protection compared with vehicle (average maximum temperature drop of $-2.577^{\circ} \mathrm{C}$ ). To determine acalabrutinib's duration of protection against anaphylaxis, engrafted NSG-SGM3 mice were pretreated with 2 doses of $15 \mathrm{mg} / \mathrm{kg}$ acalabrutinib as above, then subsequently sensitized and then challenged with $20 \mu \mathrm{g}$ NP-BSA either 2 days or 7 days after the second dose of acalabrutinib. Results show that acalabrutinib pretreatment maintained partial, though not significant, protection against moderate PSA for 2 days ( $P=0.1007$; Figure 6B), but did not impart any protection 7 days after dosing $(P=0.8342$; Figure 6C).

Acalabrutinib partially protects against fatal anaphylaxis during severe PSA in humanized mice. To test the hypothesis that acalabrutinib can protect against severe and even fatal anaphylaxis, we used the above PSA model employing a 500- $\mu$ g antigen challenge dose. Engrafted NSG-SGM3 were sensitized as above and pretreated with 2 doses of $15 \mathrm{mg} / \mathrm{kg}$ of acalabrutinib by oral gavage at 16 and 4 hours before challenge with $500 \mu \mathrm{g}$ NP-BSA. Mice pretreated with acalabrutinib had reduced clinical responses during severe PSA, with lesser core body temperature drop compared with mice pretreated with vehicle $\left(-3.232^{\circ} \mathrm{C}\right.$ vs. $-4.179^{\circ} \mathrm{C}$; $P=0.0121$; Figure 6D). Additionally, mice pretreated with acalabrutinib demonstrated a faster recovery time, beginning to show a rebound in body temperature at 40 minutes after challenge com- 
A
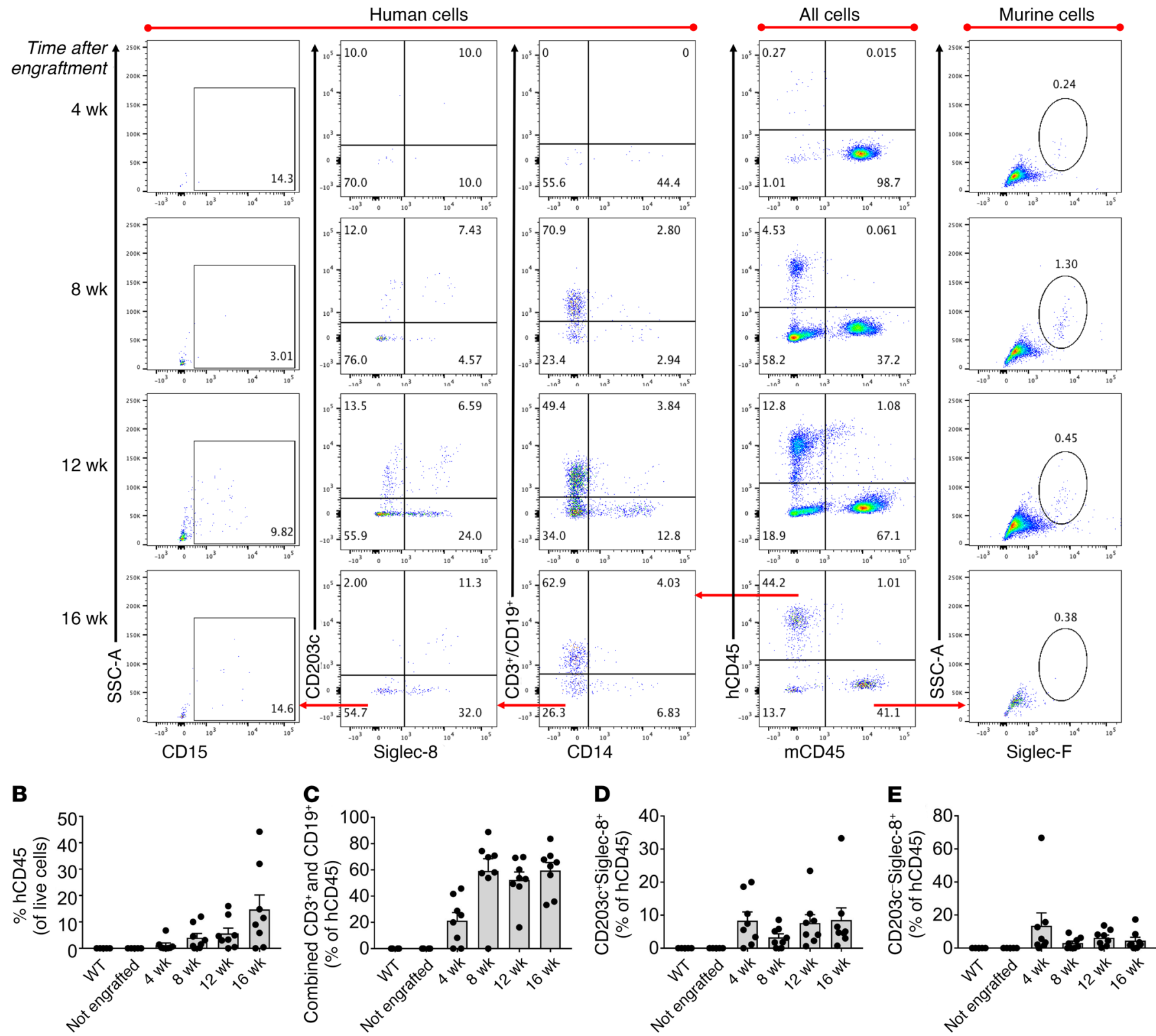

$\mathbf{F}$

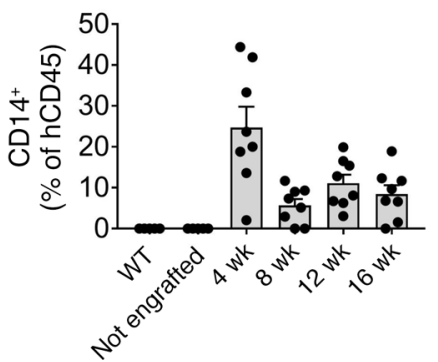

G

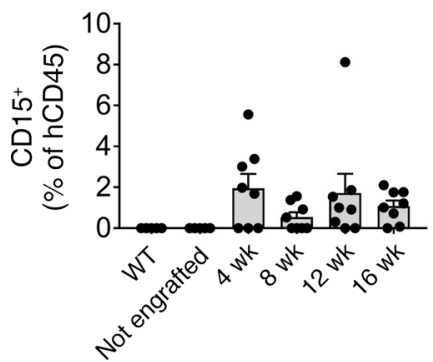

H

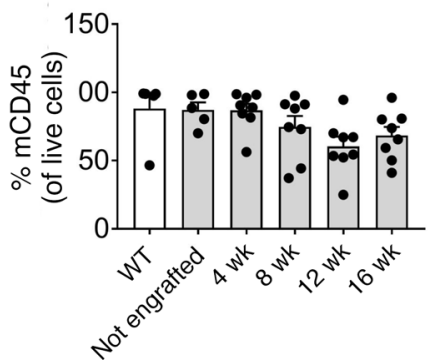

I

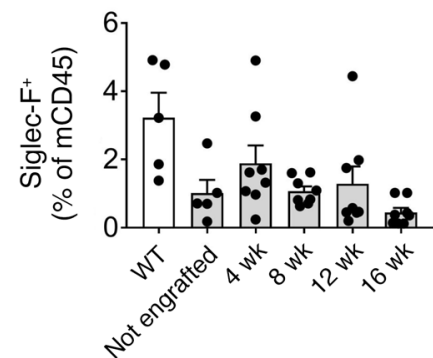

Figure 3. HSC-engrafted NSG-SGM3 mice have detectable circulating human leukocytes as early as 4 weeks after HSC injection. NSG-SCM3 mice underwent a single i.v. injection of cord blood HSCs. (A) Whole-blood samples taken at 4, 8, 12, and 16 weeks after HSC injection were incubated with fluorescently conjugated antibodies against a panel of human $(\mathrm{h})$ and murine $(\mathrm{m})$ cell surface markers as indicated and analyzed by flow cytometry. Representative flow plots from 3 separate experiments are shown for each time point. Quadrant and gate population percentages represent the percentage of the parent gate. (B-I) Percentages of cells that are human (B-C) and murine (H and I) are displayed as leukocyte subsets at the indicated time points after HSC injection; $n=5-8$ mice per group. WT mice (C57BL/6J) and non-engrafted NSG-SGM3 mice are included as controls. All data are displayed as means \pm SEM. 
A

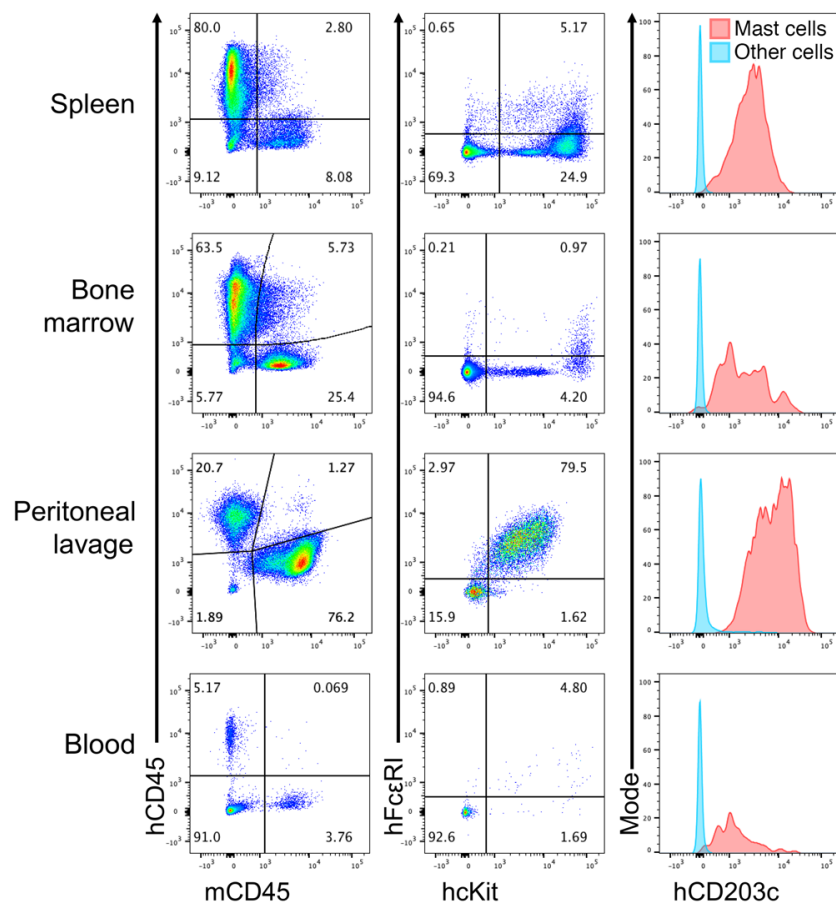

B

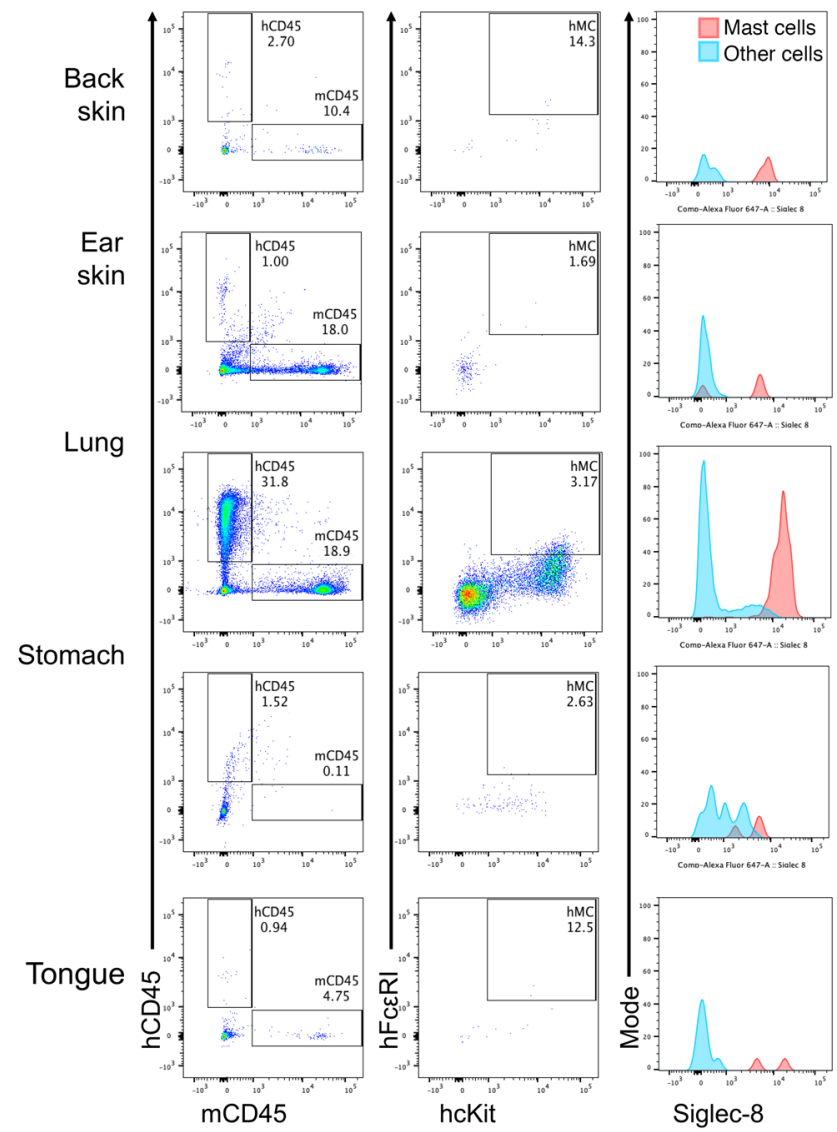

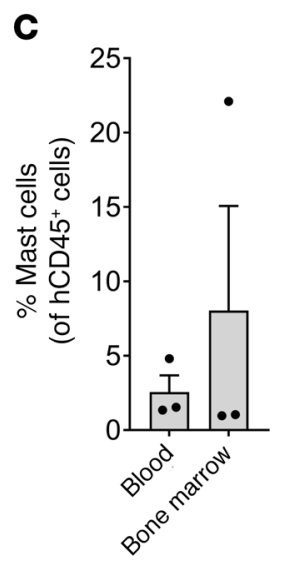
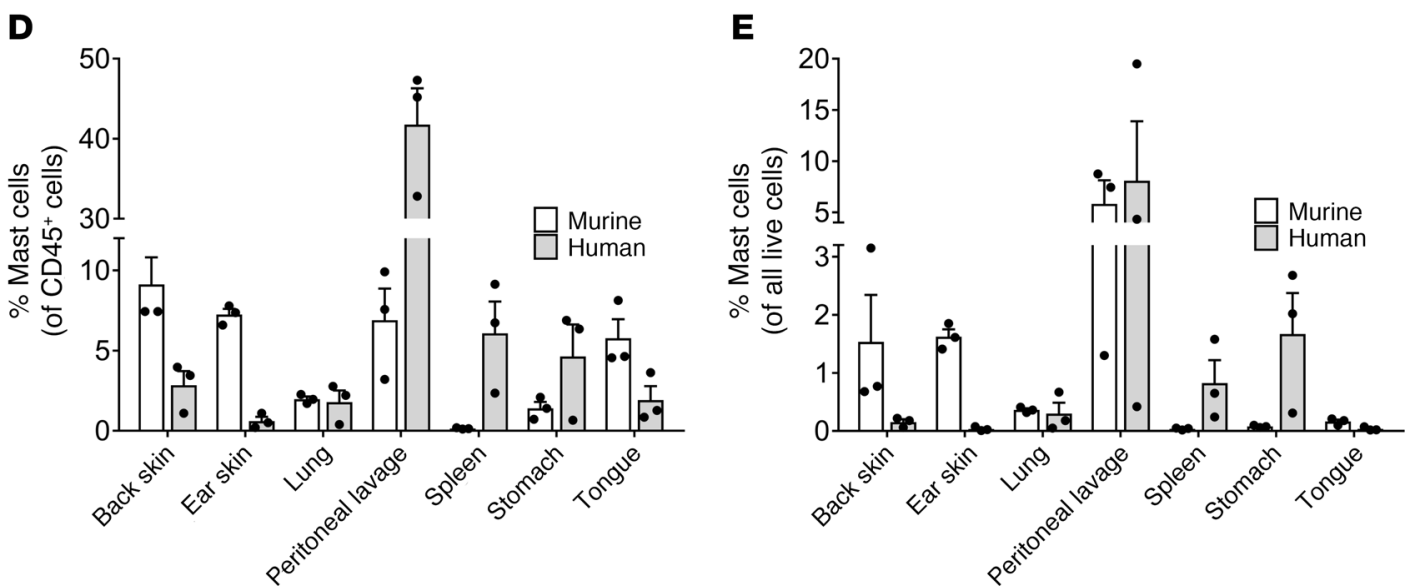

Figure 4. HSC-engrafted NSG-SGM3 mice support the accumulation, growth, and maturation of human tissue-resident mast cells. NSG-SGM3 mice underwent a single i.v. injection of cord blood HSCs. At 16 weeks after HSC injection, mice were sacrificed, and whole blood and organs were harvested and processed for detection of human and mouse mast cells and blood basophils/mast cell precursors. Single-cell suspensions were incubated with the indicated fluorescently conjugated antibodies and analyzed by flow cytometry. Cells were gated on mouse CD45 (mCD45) versus human CD45 (hCD45), and then among human cells, human $\mathrm{CKit}^{+}$(hcKit) and human $\mathrm{Fc \varepsilon RI}^{+}$(hFc\&RI) cells were analyzed for CD203c and Siglec-8 expression. Representative flow plots from 3 separate experiments are shown for spleen, bone marrow, peritoneal lavage, and whole blood (A) and for various indicated solid organs (B). Quadrant and gate population percentages represent the percentage of the parent gate. (C) Mast cell precursors were quantified as a percentage of hCD45 ${ }^{+}$ cells in blood and bone marrow; $n=3$ mice per group. ( $\mathbf{D}$ and $\mathbf{E}$ ) Mature human and murine tissue-resident mast cells were quantified as a percentage of hCD45+ or mCD45+ cells, respectively (D), and of total cells $(\mathbf{E}) ; n=3$ mice per group. All data are displayed as means \pm SEM.

pared with mice pretreated with vehicle, which began to recover at 60 minutes after challenge. Remarkably, acalabrutinib pretreatment was significantly protective against death during severe PSA, with a mortality rate of $13 \%$ compared with $39 \%$ mortality in mice pretreated with vehicle $(P=0.0365$; Figure $6 \mathrm{E})$. Collectively, these data demonstrate that 2 clinically relevant doses of acalabrutinib can rapidly and completely protect against moderate anaphylaxis and prevent death during severe anaphylaxis. 
A

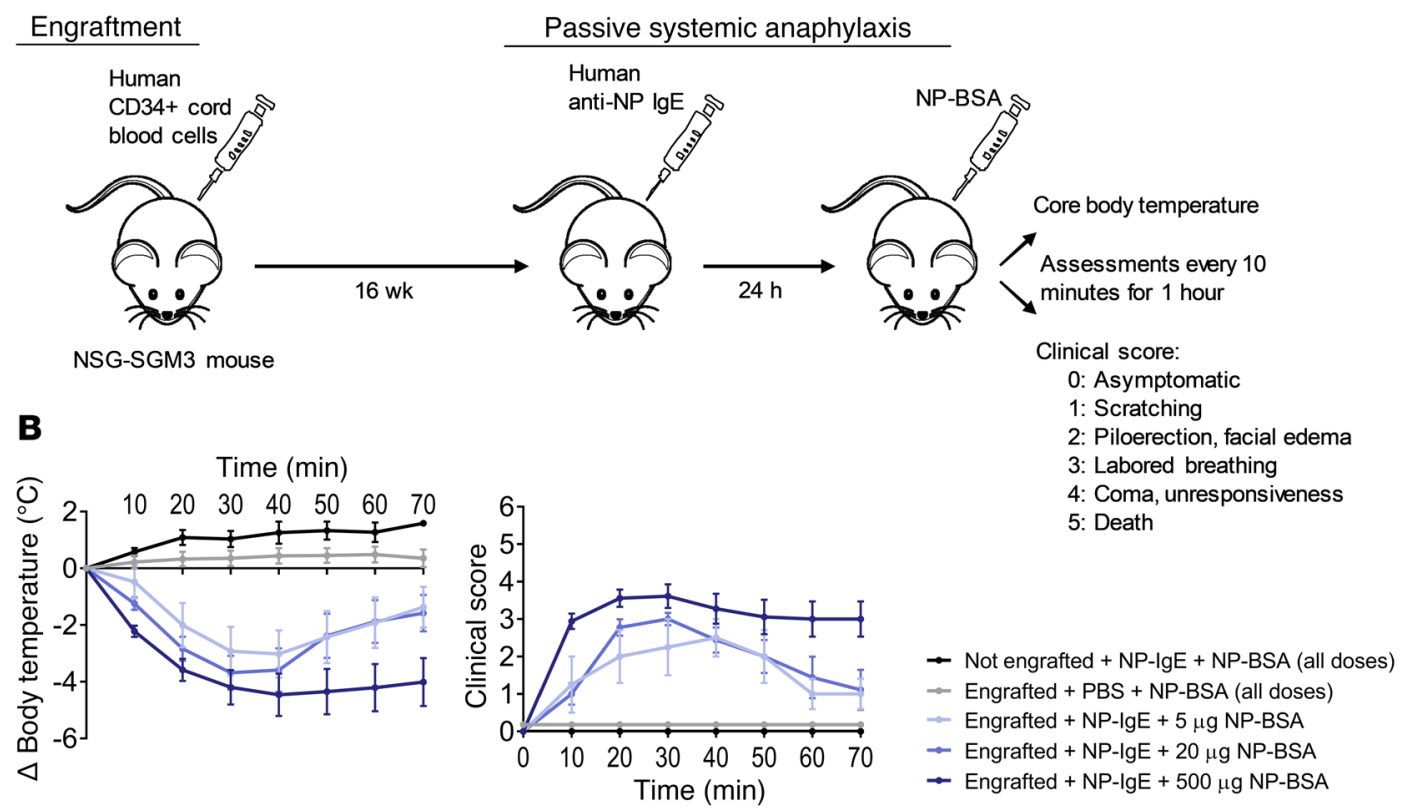

Figure 5. HSC-engrafted NSG-SGM3 mice serve as a robust model of human anaphylaxis. (A) Schema of engraftment and PSA protocol. (B) Engrafted NSG-SGM3 mice were sensitized with a single i.v. injection of $1.6 \mu \mathrm{g}$ of human anti-NP IgE and then 24 hours later were challenged with a single i.v. injection of 5, 20, or $500 \mu \mathrm{g}$ of NP-BSA to induce mild, moderate, or severe PSA, respectively. Mice sensitized with PBS instead of IgE and NSG-SGM3 mice that were not engrafted with HSCs were used as controls. The PSA response was assessed by (i) decrease in core body temperature and (ii) clinical scoring every 10 minutes for at least 1 hour after challenge. Body temperature measurements were ceased after death; therefore, only the surviving mice at each time point are included in averages. All data are displayed as means \pm SEM. $n=3-4$ mice per group.

\section{Discussion}

We herein show complete clinical protection from moderateseverity IgE-mediated anaphylaxis using pretreatment with just 2 oral doses of the BTKi acalabrutinib in what we believe to be a novel, simplified, less costly, and more humane animal model for PSA. With irradiation and/or chemotherapy ablation of the bone marrow plus surgical implantation of human fetal liver and thymic tissues, prior models using NSG-SGM3 have proven to be valuable for hypersensitivity disorders and PSA as has been demonstrated in prior studies $(24,25,28,29)$. Our data here demonstrate that NSG-SGM3 mice can still adequately engraft human leukocytes after a single i.v. injection of HSCs without survival surgery or prior bone marrow ablation. To our knowledge, this is the first demonstration of this model's ability to support the growth of human tissue-resident mast cells and its utility for performing PSA experiments. Analysis of whole blood and organ tissues displayed populations of both $\mathrm{hCD} 45^{+}$and $\mathrm{mCD} 45^{+}$cells after HSC injection, indicating that human HSCs can engraft alongside murine cells in the murine bone marrow and proliferate to create mature human leukocytes (Figures 3 and 4). Additionally, data demonstrated the presence of mature human tissue-resident mast cells in the organs of engrafted NSG-SGM3 mice (Figure 4). Therefore, the presence of human liver and/or thymic tissue is not necessary for human mast cell growth or maturation (24).

As NSG-SGM3 mice constitutively express serum levels of SCF that are comparable to those found in healthy humans $(23,30)$, the human mast cells in these mice are unlikely to be phenotypically hyperproliferative or overactive, but this was not specifically analyzed. We did, however, find that the organ distribution of human mast cells in this model differed from that of murine mast cells. Specifically, human mast cells far outnumbered murine mast cells in the peritoneal cavity, spleen, and stomach, but were relatively sparse in the skin. This altered tissue distribution, in addition to species differences in human mast cell versus murine mast cell mediator content and other factors, may alter the physiologic anaphylactic response during PSA in humanized mice compared with WT mice. For example, the lack of human mast cells in the skin may have reduced the pruritus and scratching behavior that are typically observed during PSA in WT mice. Nonetheless, by variation of the amount of NP-BSA used for challenge in this model, PSA severity and mortality rate can be titrated, allowing for control over the severity of the anaphylactic response (Figure 5). This allowed us to test the ability of BTKis to prevent mild to moderate anaphylaxis as well as fatal anaphylaxis, which cannot be ethically tested in humans. Despite the presence of mature murine tissue-resident mast cells, non-engrafted NSG-SGM3 mice did not show any anaphylactic response to sensitization with human IgE, in line with previously published reports that human IgE cannot bind to murine FceRI receptors (26). Thus, the presence of circulating murine leukocytes does not appear to influence passive anaphylaxis experiments using human IgE, and ablation of the bone marrow before engraftment is not necessary for adequate engraftment of human HSCs. It should be noted that although NSG mice (which do not have human cytokine transgenes) can still mount human antibody responses to antigen without prior surgical implantation of human tissues (31), the addition of human tissues is thought to augment the maturation 
A
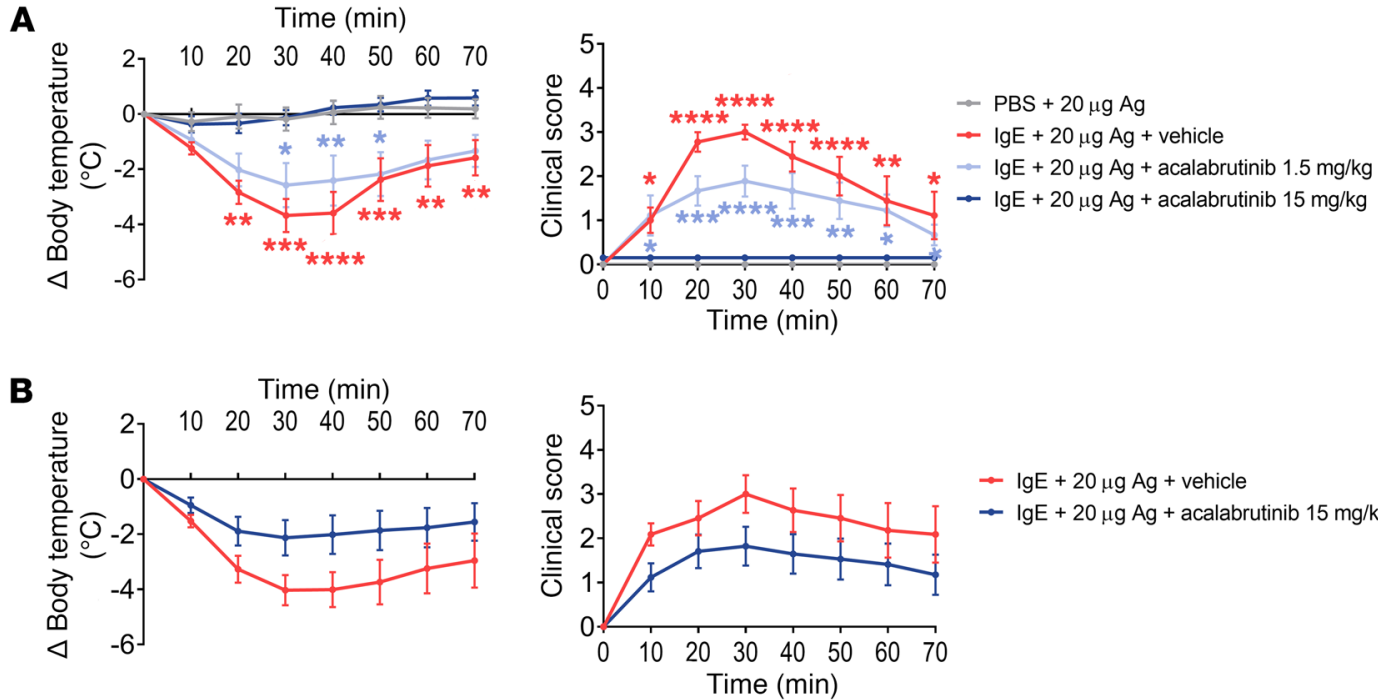

$$
\begin{aligned}
& \rightarrow \operatorname{lgE}+20 \mu \mathrm{g} \mathrm{Ag}+\text { vehicle } \\
& \rightarrow \operatorname{lgE}+20 \mu \mathrm{g} \mathrm{Ag}+\text { acalabrutinib } 15 \mathrm{mg} / \mathrm{kg}
\end{aligned}
$$
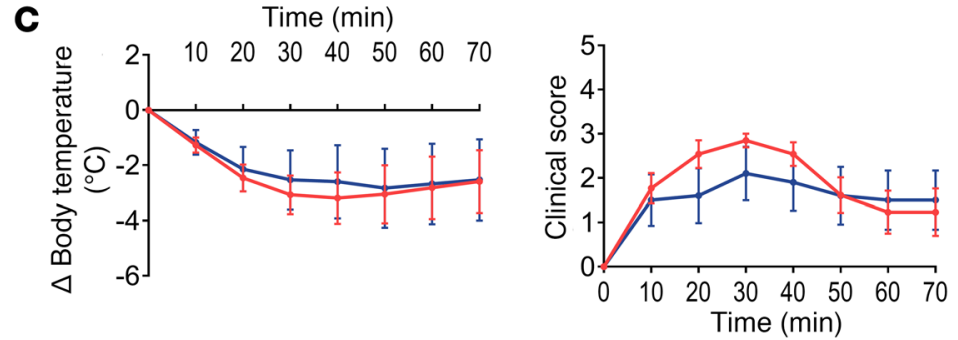

$\rightarrow \lg \mathrm{E}+20 \mu \mathrm{g} \mathrm{Ag}+$ vehicle

$\rightarrow \operatorname{lgE}+20 \mu \mathrm{g} \mathrm{Ag}+$ acalabrutinib $15 \mathrm{mg} / \mathrm{kg}$
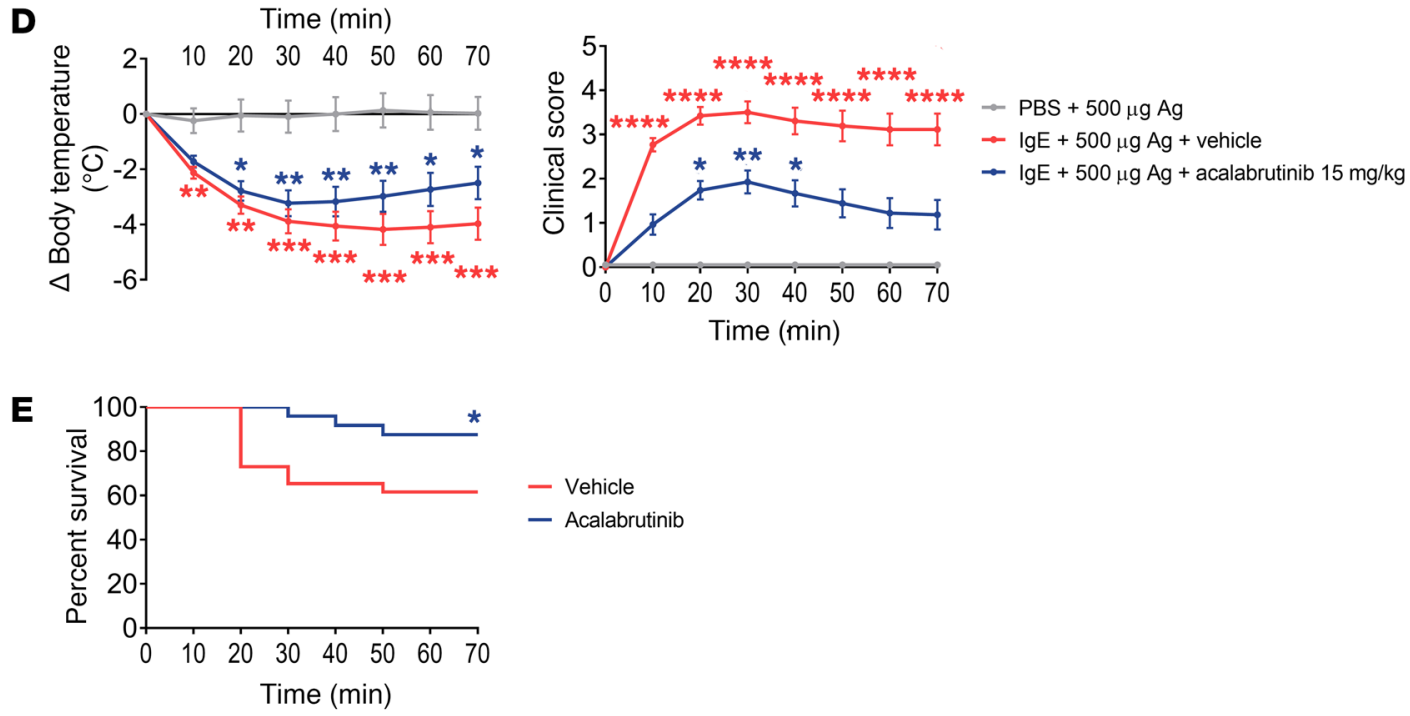

Figure 6. Acalabrutinib pretreatment completely inhibits moderate PSA and partially protects against fatal PSA in humanized mice. (A) HSC-engrafted humanized mice were sensitized and then pretreated with 2 doses of acalabrutinib $(1.5 \mathrm{or} 15 \mathrm{mg} / \mathrm{kg}$ via gavage) or vehicle control at 16 and 4 hours before challenge with $20 \mu \mathrm{g} \mathrm{NP-BSA}$ to elicit moderate PSA. Core temperature drop from baseline (left) and clinical scores (right) are shown as measures of clinical response during PSA. Body temperature measurements were ceased after death; therefore, only the surviving mice at each time point are included in averages. Data are pooled from 3 independent experiments; $n=6-9$ total per group. (B and $\mathbf{C}$ ) To investigate the duration of acalabrutinib's protection, engrafted humanized mice were sensitized and pretreated with acalabrutinib as described in A, except that NP-BSA challenge was performed either 2 days (B) or 7 days (C) after the last oral dose of acalabrutinib. Data are pooled from 3 separate experiments; $n=11-17$ total per group. (D) To investigate acalabrutinib's ability to prevent fatal anaphylaxis, engrafted humanized mice were sensitized and pretreated with $15 \mathrm{mg} / \mathrm{kg}$ acalabrutinib or vehicle, except a higher challenge dose ( $500 \mu \mathrm{g}$ NP-BSA) was given to elicit a more severe PSA response. Data shown are pooled from 6 separate experiments; $N=$ 24-27 total per group. (E) The Kaplan-Meier survival curve from experiments in $\mathbf{D}$ is shown for both the acalabrutinib-and vehicle-treated groups. All data were analyzed using 2-way ANOVA with repeated measures with the exception of the mortality rate in $\mathbf{E}$, which was analyzed using $\chi^{2}$ analysis. All data are displayed as means \pm SEM. ${ }^{*} P<0.05,{ }^{* *} P<0.01,{ }^{* *} P<0.001,{ }^{* * * *} P<0.0001$ compared with PBS control group. 
of human lymphocytes in other humanized mouse models (32). Therefore, it is unknown whether the herein-described model of humanized NSG-SGM3 mice could also be used for allergen sensitization models, but this seems unlikely.

Current therapies for treating allergic responses are restricted to blocking signs and symptoms caused by mediators such as histamine and leukotrienes, which are just a few of the many mediators released by activated mast cells and basophils. No known therapies are capable of preventing anaphylaxis, including antihistamines, because mediators other than histamine are involved in severe reactions including hypotension and shock (33). Therefore, targeting all IgE-mediated signaling through the FceRI pathway upstream of mediator release would be an optimal approach for preventing anaphylaxis to any antigen. The FceRI pathway includes multiple kinases other than BTK, including Syk, Lyn, Fyn, PI3K, and others. As a first-generation BTKi, ibrutinib has several off-target effects, including inhibition of Lyn and Fyn in the IgE pathway (34). Other off-target effects, such as activity on the epidermal growth factor receptor (EGFR) and tyrosine kinase expressed in hepatocellular carcinoma (TEC), may be beneficial in correcting underlying immune dysregulation in cancers such as CLL, but also likely account for a large portion of ibrutinib's toxicity and side effect profile (9). Because EGFR and TEC kinase are not known to be involved in FceRI signaling, activity on these kinases is likely irrelevant for protection from anaphylaxis and even undesired from a clinical standpoint. In line with this hypothesis, we have shown that acalabrutinib and tirabrutinib, second-generation inhibitors with far fewer off-target effects than ibrutinib $(35,36)$, are equally as effective as ibrutinib in preventing IgE-mediated activation of primary human mast cells and basophils (Figure 1). Therefore, it is evident that ibrutinib's off-target effects are not necessary for its prevention of FceRI signaling and that BTK is the true pharmacologic target.

Though additional compounds designed to target other kinases in the FceRI signaling pathway have also shown efficacy in preventing anaphylaxis in murine models (37-44), none of them have also achieved FDA approval owing to their toxicity, which is due to the broad expression of their target kinases in numerous tissues and cell types. In contrast, BTK is expressed primarily in leukocytes, including B cells, mast cells, basophils, macrophages, and neutrophils (45). Congenital BTK deficiency (X-linked agammaglobulinemia [XLA]) is characterized by low or absent circulating $\mathrm{B}$ cells and a lack of humoral immune responses (46). However, pharmacologic inhibition of BTK is phenotypically different, presumably because B cell maturation largely occurs before a BTKi is used clinically. FDA-approved BTKis are generally well tolerated, though serious side effects such as infection, bone marrow suppression, bleeding, and arrhythmia are reported with chronic use in cancer patients $(8-10,47)$. Several new BTKis in development are in clinical trials for other chronic disorders such as autoimmune diseases and urticaria (48), suggesting that their safety profiles during chronic use may be acceptable for these non-cancer indications. Regardless, the safety profiles of second-generation BTKis appear favorable for short-term courses, as would be the case for prophylactic use. We were the first to publish safety data for the short-term use of a BTKi in healthy adults without cancer: administration of up to 7 daily oral doses of $420 \mathrm{mg}$ of ibrutinib was well tolerated and showed no detectable toxicity in healthy adults with food allergy (11).

Collectively, our data suggest that acalabrutinib pretreatment is remarkably protective against IgE-mediated human mast cell secretion in vitro and anaphylaxis in humanized mice in vivo, with a rapid onset and relatively short duration of action once discontinued. As a covalent inhibitor of BTK, acalabrutinib displays prolonged inhibitory effects in vivo despite a relatively short serum half-life of roughly 1 hour $(35,49,50)$. Owing to pharmacokinetic differences between mice and humans, $15-\mathrm{mg} / \mathrm{kg}$ oral dosing in mice and the FDA-approved 100-mg dose in humans both result in nearly $100 \%$ BTK occupancy in splenocytes as soon as 3 hours, and up to 24 hours, after a single dose (35). Interestingly, our data showed that partial protection from anaphylaxis was achieved with $1.5 \mathrm{mg} / \mathrm{kg}$ dosing, which corresponds to roughly $50 \% \mathrm{BTK}$ occupancy in splenocytes in mice, demonstrating that partial clinical protection can be achieved even without full BTK inhibition. Thus, our data show that 2 clinically relevant oral doses of acalabrutinib completely prevented moderate anaphylaxis and significantly protected against death during severe anaphylaxis (Figure 6). This protection was only partially sustained for 2 days after the last dose, demonstrating that the effects of irreversible BTKis on prevention of anaphylaxis are temporary. While not specifically studied, the kinetics of recovery provides some insight into the rate of turnover of BTK in mast cells, given that the only logical way for these irreversible inhibitors to lose their activity is via cell proliferation or synthesis of new BTK in the absence of drug. Furthermore, the transience of the effects of BTKis may be beneficial in terms of their safety profile, especially when they are used intermittently to prevent anaphylaxis. For instance, it may be possible to episodically use short courses of BTKis to prevent IgE-mediated reactions including anaphylaxis during a number of clinical procedures such as rush food or venom immunotherapy or drug desensitizations.

The choice to use 2 doses of acalabrutinib in the animal models was drawn from our prior work showing that 2 FDA-approved doses of $420 \mathrm{mg}$ of ibrutinib markedly reduces food skin prick test size in food-allergic adults (11). Prior studies have shown that a single FDA-approved oral dose of ibrutinib or acalabrutinib achieves nearly $100 \%$ BTK occupancy in peripheral blood mononuclear cells within just 3-4 hours $(14,35)$. While we did not investigate the efficacy of 1 dose of acalabrutinib in humanized mice, it may be possible that 1 dose would be sufficient to protect against anaphylaxis. The penetrance of BTKis into various tissues where mast cells reside is unknown. We and others have demonstrated their rapid efficacy in preventing IgE-mediated activation of circulating basophils $(6,11,13,14)$, but it is unknown whether these drugs would fully inhibit IgE-mediated activation of tissue-resident mast cells in multiple organs such as the lung, skin, and gastrointestinal tract.

In conclusion, selective inhibitors of BTK can prevent IgEmediated degranulation and cytokine production in human mast cells and prevent IgE-mediated anaphylaxis in humanized mice. To our knowledge, this is the first report of an oral drug's ability to completely prevent human mast cell-mediated anaphylaxis. A logical next step for future studies would be to examine the safety and efficacy of BTKis in preventing anaphylaxis in humans. If effective, these drugs may have the potentially paradigm-changing 
property of being capable of preventing systemic allergic reactions, including fatal anaphylaxis, in an allergen-independent manner.

\section{Methods}

Human SDMCs. Cultures of skin-derived mast cells (SDMCs) were prepared as previously described (16). In brief, discarded surgical samples of human skin were obtained from deidentified sources through the Cooperative Human Tissue Network (supported by the National Cancer Institute, NIH). Skin fragments were mechanically minced and then processed via enzymatic digestion with Collagenase Type 2 (Worthington Biochemical Corp.), DNase Type 1 (MilliporeSigma), and hyaluronidase (MilliporeSigma) before isolation of mononuclear cells via centrifugation with Percoll PLUS (GE Healthcare). Single-cell suspensions were cultured for 4 weeks in X-Vivo15 Serum-Free Culture Medium (Lonza) containing $100 \mathrm{ng} / \mathrm{mL}$ recombinant human stem cell factor (SCF; PeproTech). Culture purity was assessed via flow cytometry for cKit and FceRI expression.

Degranulation and cytokine assays. Lyophilized stocks of the BTKis ibrutinib (Selleckchem), acalabrutinib (provided by Acerta Pharma, a member of the AstraZeneca Group, San Francisco, California, USA), and tirabrutinib (Selleckchem) were resuspended in DMSO and stored at $-80^{\circ} \mathrm{C}$. Fresh dilutions in sterile PBS were made from stocks for each experiment. Percentage of total $\beta$-hexosaminidase release from SDMCs was determined using colorimetric assay as previously described (51). In brief, cells were passively sensitized overnight with $50 \mathrm{ng} / \mathrm{mL}$ biotinylated human IgE (Abbiotec, clone HE1) in culture media. The next day, cells were treated with BTKis for 15 minutes at $37^{\circ} \mathrm{C}$ at the indicated time points, washed 3 times, and then resuspended in Tyrode's buffer with $100 \mathrm{ng} / \mathrm{mL}$ streptavidin (MilliporeSigma) at $37^{\circ} \mathrm{C}$ for 1 hour to cross-link IgE. Supernatants and corresponding cell lysates were incubated with $1.37 \mathrm{mg} / \mathrm{mL} 4$-nitrophenyl $N$-acetyl- $\beta$-D-glucosaminide at $37^{\circ} \mathrm{C}$ for 1 hour. The reaction was stopped with carbonate buffer, and absorbance was read at $405 \mathrm{~nm}$ on a plate reader. Percentage of total $\beta$-hexosaminidase release was calculated. To detect surface marker upregulation, SDMCs were sensitized with IgE, treated with BTKis, and activated using streptavidin as above. One hour after activation, tubes were placed on ice for 5 minutes to stop reactions. Cells were labeled with fluorescently conjugated antibodies (Supplemental Table 1) and analyzed by flow cytometry using an LSR II cytometer (Becton Dickinson) and FACSDiva acquisition software (BD Biosciences). Flow cytometry data were analyzed using FlowJo (v10) software (Becton Dickinson). Cells were first gated on scatter and $\mathrm{cKit}^{+}$. LAMP1 upregulation was determined by the percentage of $\mathrm{cKit}^{+}$cells that were LAMP1 ${ }^{+}$. CD203c upregulation was determined by mean MFI. For cytokine release assays, cells were sensitized as above and treated with $100 \mathrm{ng} / \mathrm{mL}$ streptavidin in media for 24 hours at $37^{\circ} \mathrm{C}$. Supernatants were collected and analyzed for cytokine content by fluorescent multiplex assay (MilliplexMAP, Millipore) and read on a Luminex 200 plate reader. Cytokine concentrations were calculated according to the manufacturer's directions.

Basophil activation testing. Peripheral whole-blood samples were obtained via phlebotomy from healthy adults and collected in EDTA vacutainer tubes (Becton Dickinson). Basophil activation testing was performed using Flow CAST Basophil Activation kits (Buhlmann Diagnostics). Whole-blood samples were incubated with BTKis for 15 minutes at $37^{\circ} \mathrm{C}$ before activation per kit directions with anti-FceRI $\alpha$ antibody or fMLP as a control. Samples were then incubated with fluorescently labeled antibodies and analyzed by flow cytometry. All cells were gated based on light scatter and CCR3 expression to gate on basophils, and the percentage of activated basophils was calculated using CD63 surface upregulation.

Bronchial contraction assays. Anti-IgE-induced increases in isomeric tension of isolated human bronchi were measured as described previously (17). Briefly, intralobar bronchi (2-5 mm diameter) were dissected from deidentified normal human donor lung tissue provided | by the International Institutes for the Advancement of Medicine. To measure isometric tension, tissues were connected to Grass FTO3 force transducers, and tension was recorded on a Grass Model 7 polygraph (Grass Instruments). Isolated bronchi were suspended in $10 \mathrm{~mL}$ water-jacketed tissue baths containing Krebs bicarbonate buffer solution and gassed with oxygen/carbon dioxide $(95: 5)$ at $37^{\circ} \mathrm{C}$. Tissues were maintained at a resting tension of $1 \mathrm{~g}$ for a 2-hour equilibration period, during which the buffer was replaced at 15-minute intervals. After a 30-minute incubation with either vehicle or ibrutinib, increasing concentrations of polyclonal goat anti-human IgE (created and purified as previously described, ref. 52; provided by Donald MacGlashan Jr., Johns Hopkins University, Baltimore, Maryland, USA) or histamine were added to the tissue bath until bronchial contraction reached a steady state (10-15 minutes), at which time the next concentration of stimulant was added. This was continued until maximal anti-IgE- or histamine-induced contraction was obtained, and cumulative concentration-response curves for contraction were calculated. Contractions in response to anti-IgE and histamine were normalized to the maximal tissue contraction as evoked by $100 \mu \mathrm{M}$ carbamylcholine added to the same tissues after the maximal histamine or anti-IgE response was obtained.

Humanized mice. Female NOD.Cg-Prkdc scid Il2rgtmiwjl $\mathrm{Tg}(\mathrm{CMV}-I L 3, C S F 2, K I T L G) 1 \mathrm{Eav}$ mice ("NSG-SGM3") were purchased from The Jackson Laboratory. Humanized mice were created by i.v. injection at age $3-4$ weeks with $5 \times 10^{5}$ commercially purchased human cord blood CD34 ${ }^{+}$stem cells (HSCs; Lonza). To characterize the model, whole blood was collected from engrafted mice via retroorbital bleed into microtainer EDTA tubes (Becton Dickinson) at 4, 8,12 , and 16 weeks after injection with HSCs. For analysis of cells in tissues, engrafted mice were sacrificed 16 weeks after HSC injection, and peritoneal lavage and indicated organs were collected immediately after sacrifice. Spleens were compressed between the frosted surface of 2 glass slides to obtain single-cell suspensions. Ear and skin tissue digestion was performed as previously published $(53,54)$. Briefly, hair was removed and skin tissue was placed in HBSS with $2 \mathrm{U} / \mathrm{mL}$ of freshly prepared Liberase TM. Ventral and dorsal halves were split and minced with scissors. Tissue was then digested in conical tubes for 30 minutes at $37^{\circ} \mathrm{C}$ with agitation at $250 \mathrm{rpm}$ in an orbital shaker. Digestion was stopped with cold PBS containing $15 \%$ FBS and strained through a $70-$ to $100-\mu \mathrm{m}$ strainer. Lung, stomach, and tongue tissue was digested as previously described (55). Tissues were minced and placed in digestion buffer (RPMI containing 10\% FBS, $600 \mathrm{U} / \mathrm{mL}$ collagenase IV [Worthington Biochemical Corp.], $0.1 \%$ dispase [Gibco], and $20 \mu \mathrm{g} / \mathrm{mL}$ DNase I [Roche]) for $30 \mathrm{~min}$ utes at $37^{\circ} \mathrm{C}$ with agitation at $300 \mathrm{rpm}$ in an orbital shaker. The digestion was quenched with FBS, and homogenates were strained as above. In all samples, red blood cells were lysed using RBC lysis buffer (eBioscience), and samples were blocked using rat anti-mouse 
CD16/CD32 (BD Biosciences), anti-human CD32 (BioLegend), and True Stain Monocyte Blocker (BioLegend). Samples were then stained with fluorescently conjugated antibodies (see complete list in Supplemental Table 1) and Aqua Fluorescent Reactive Dye (Invitrogen) and run by flow cytometry on an LSR II cytometer (BD Life Sciences) using FACSDiva software (BD Life Sciences). Data were analyzed using FlowJo v10 software (BD Life Sciences). Gating strategies are shown in Supplemental Figures 2 and 3. As data demonstrated that the 12- and 16-week bleeds showed equivalent results, engrafted mice used for subsequent experiments with acalabrutinib were checked for adequate human leukocyte engraftment once at 12 weeks after HSC injection to allow for a period of recovery before PSA experiments. Based on the lack of anaphylactic response in mice with poor engraftment (data not shown), mice were considered to have adequate engraftment for use in future PSA experiments if at least $0.1 \%$ of total $C D 45^{+}$circulating cells were human $C D 45^{+}$at 12 weeks after HSC injection. All mice were housed in barrier housing, and received enrofloxacin in their drinking water as prophylaxis against Corynebacterium bovis.

Passive systemic anaphylaxis. PSA experiments were performed starting 16 weeks after HSC injection. Human chimeric IgE specific to 4-hydroxy-3-nitrophenylacetyl (NP) was harvested from serumfree media of JW8/5/13 hybridoma cell cultures (European Collection of Authenticated Cell Cultures), purified by density centrifugation, and quantified using a human IgE ELISA. PSA was performed using a previously published protocol (24): mice were anesthetized with a ketamine/xylazine cocktail and passively sensitized with an i.v. injection of $1.6 \mu \mathrm{g}$ human NP-IgE in $100 \mu \mathrm{L}$ PBS (or PBS vehicle) 24 hours before i.v. challenge with various doses of NP-BSA conjugate (Biosearch LGC) in PBS. Early-phase PSA response was assessed using core body temperature by rectal probe and visual clinical scoring as previously described (56) immediately after challenge, every 10 minutes for at least 60 minutes or until full recovery. In brief, clinical scores were 1 (scratching), 2 (piloerection, facial edema), 3 (labored breathing), 4 (coma or unresponsiveness), and 5 (death). When PSA induced a fatal response, body temperature measurements were ceased. Mice were not reused for repeat PSA experiments.

Treatment with acalabrutinib. Lyophilized acalabrutinib (ACP196, Acerta Pharma) was resuspended in a solution of 0.5\% methylcellulose (MilliporeSigma)/0.1\% Tween 80 (MilliporeSigma) as recommended by the manufacturer. Resuspensions of acalabrutinib stock were made fresh for each PSA experiment. Mice were treated with vehicle or acalabrutinib at the indicated doses via oral gavage at the indicated time points before NP-BSA antigen challenge.

Statistics. Data are given as means \pm SEM unless otherwise noted. Data were analyzed using 2-way ANOVA with repeated measures by Dunnett's multiple-comparisons test with a family-wise significance rate of 0.05. Exceptions include the comparison of mortality rates between acalabrutinib and vehicle-treated groups during severe anaphylaxis, which were analyzed using $\chi^{2}$ analysis, and the total contraction from carbamylcholine treatment and percentage of maximal contraction from histamine treatment of human bronchi, which were analyzed using 2-tailed paired Student's $t$ tests. For all statistics, a $P$ value of less than 0.05 was considered significant. All analyses were done in GraphPad Prism software v7.05.

Study approval. Deidentified normal human lung and skin fragments were obtained under an IRB waiver from sources described above. Human whole-blood samples were obtained from healthy donors under a protocol approved by Northwestern University's Institutional Review Board. All animal studies were conducted under the approval of Northwestern University's Institutional Animal Care and Use Committee.

\section{Author contributions}

MCD designed and conducted experiments, analyzed data, and wrote the manuscript. RAKB, KDC, PAR, and BJU performed experiments and analyzed data. BSB designed experiments and edited the manuscript.

\section{Acknowledgments}

The authors would like to convey their immense gratitude to Allard Kaptein, Cecile Krejsa, Diana Mittag, Todd Covey, and Tjeerd Barf at Acerta Pharma (a member of the AstraZeneca Group) for their helpful discussions, guidance, and support. This work was funded by Acerta Pharma, the Ernest S. Bazley Foundation, and the Northwestern University Allergy Immunology Research Program T32 AI083216 (National Institute of Allergy and Infectious Diseases).

Address correspondence to: Bruce S. Bochner, Division of Allergy and Immunology, Department of Medicine, Northwestern University Feinberg School of Medicine, 240 East Huron Street, Room M-306, Chicago, Illinois 60611, USA. Phone: 312.503.0068; Email: bruce.bochner@northwestern.edu.
1. Shaker MS, et al. Anaphylaxis-a 2020 practice parameter update, systematic review, and Grading of Recommendations, Assessment, Development and Evaluation (GRADE) analysis. J Allergy Clin Immunol. 2020;145(4):1082-1123.

2. Lieberman P, et al. Epidemiology of anaphylaxis: findings of the American College of Allergy, Asthma and Immunology Epidemiology of Anaphylaxis Working Group. Ann Allergy Asthma Immunol. 2006;97(5):596-602.

3. Liu A, et al. Desensitization regimens for drug allergy: state of the art in the 21st century. Clin Exp Allergy. 2011;41(12):1679-1689.

4. Iyer AS, et al. Absence of Tec family kinases interleukin-2 inducible T cell kinase (Itk) and Bruton's tyrosine kinase (Btk) severely impairs Fc $\epsilon \mathrm{RI}$-dependent mast cell responses. J Biol Chem.
2011;286(11):9503-9513.

5. Kuehn HS, Swindle EJ, Kim MS, Beaven MA, Metcalfe DD, Gilfillan AM. The phosphoinositide 3-kinase-dependent activation of Btk is required for optimal eicosanoid production and generation of reactive oxygen species in antigen-stimulated mast cells. J Immunol. 2008;181(11):7706-7712.

6. MacGlashan D, Honigberg LA, Smith A, Buggy J, Schroeder JT. Inhibition of IgE-mediated secretion from human basophils with a highly selective Bruton's tyrosine kinase, Btk, inhibitor. Int Immunopharmacol. 2011;11(4):475-479.

7. Hendriks RW, Yuvaraj S, Kil LP. Targeting Bruton's tyrosine kinase in B cell malignancies. Nat Rev Cancer. 2014;14(4):219-232.

8. Burger JA. Bruton tyrosine kinase inhibitors: present and future. Cancer J. 2019;25(6):386-393.
9. Lucas F, Woyach JA. Inhibiting Bruton's tyrosine kinase in CLL and other B-cell malignancies. Target Oncol. 2019;14(2):125-138.

10. Hanna KS, Campbell M, Husak A, Sturm S. The role of Bruton's tyrosine kinase inhibitors in the management of mantle cell lymphoma [published online April 11, 2020]. J Oncol Pharm Pract. https://doi.org/10.1177/1078155220915956.

11. Dispenza MC, Pongracic JA, Singh AM, Bochner BS. Short-term ibrutinib therapy suppresses skin test responses and eliminates IgE-mediated basophil activation in adults with peanut or tree nut allergy. J Allergy Clin Immunol. 2018;141(5):1914-1916.e7.

12. Regan JA, et al. Ibrutinib, a Bruton's tyrosine kinase inhibitor used for treatment of lymphoproliferative disorders, eliminates both aeroallergen 
skin test and basophil activation test reactivity. J Allergy Clin Immunol. 2017;140(3):875-879.e1.

13. Smiljkovic D, et al. BTK inhibition is a potent approach to block IgE-mediated histamine release in human basophils. Allergy. 2017;72(11):1666-1676.

14. Advani RH, et al. Bruton tyrosine kinase inhibitor ibrutinib (PCI-32765) has significant activity in patients with relapsed/refractory B-cell malignancies. JClin Oncol. 2013;31(1):88-94.

15. Gamperl S, et al. Effects of ibrutinib on proliferation and histamine release in canine neoplastic mast cells. Vet Comp Oncol. 2019;17(4):553-561.

16. Kambe N, Kambe M, Kochan JP, Schwartz LB. Human skin-derived mast cells can proliferate while retaining their characteristic functional and protease phenotypes. Blood. 2001;97(7):2045-2052.

17. Ellis JL, Hubbard WC, Meeker S, Undem BJ. Ragweed antigen $\mathrm{E}$ and anti-IgE in human central versus peripheral isolated bronchi. Am J Respir Crit Care Med.1994;150(3):717-723.

18. Hata D, et al. Involvement of Bruton's tyrosine kinase in FceRI-dependent mast cell degranulation and cytokine production. JExp Med. 1998;187(8):1235-1247.

19. Chang BY, et al. The Bruton tyrosine kinase inhibitor PCI-32765 ameliorates autoimmune arthritis by inhibition of multiple effector cells. Arthritis Res Ther. 2011;13(4):R115.

20. Regan JA, Krier-Burris R, O'Sullivan J, Bryce P, Bochner BS. Pretreatment with ibrutinib, a Bruton's tyrosine kinase inhibitor, reduces passive systemic anaphylaxis in a murine model. JAllergy Clin Immun. 2016;137(2):AB50.

21. Billerbeck E, Barry WT, Mu K, Dorner M, Rice CM, Ploss A. Development of human CD4 FoxP3+ regulatory $\mathrm{T}$ cells in human stem cell factor-, granulocyte-macrophage colonystimulating factor-, and interleukin-3-expressing NOD-SCID IL2R $\gamma$ (null) humanized mice. Blood. 2011;117(11):3076-3086.

22. Coughlan AM, et al. Myeloid engraftment in humanized mice: impact of granulocyte-colony stimulating factor treatment and transgenic mouse strain. Stem Cells Dev. 2016;25(7):530-541.

23. Nicolini FE, Cashman JD, Hogge DE, Humphries RK, Eaves CJ. NOD/SCID mice engineered to express human IL-3, GM-CSF and Steel factor constitutively mobilize engrafted human progenitors and compromise human stem cell regeneration. Leukemia. 2004;18(2):341-347.

24. Bryce PJ, et al. Humanized mouse model of mast cell-mediated passive cutaneous anaphylaxis and passive systemic anaphylaxis. JAllergy Clin Immunol. 2016;138(3):769-779.

25. Youngblood BA, et al. AKOO2, a humanized sialic acid-binding immunoglobulin-like lectin-8 antibody that induces antibody-dependent cell-mediated cytotoxicity against human eosinophils and inhibits mast cell-mediated anaphylaxis in mice. Int Arch Allergy Immunol. 2019;180(2):91-102.
26. Kraft S, Kinet JP. New developments in FceRI regulation, function and inhibition. Nat Rev Immunol. 2007;7(5):365-378.

27. Dispenza MC, Krier-Burris RA, Bochner BS. The Bruton's tyrosine kinase inhibitor acalabrutinib protects from anaphylaxis in a humanized mouse model. J Allergy Clin Immun. 2019;143(2):AB144.

28. Burton OT, Stranks AJ, Tamayo JM, Koleoglou KJ, Schwartz LB, Oettgen HC. A humanized mouse model of anaphylactic peanut allergy. J Allergy Clin Immunol. 2017;139(1):314-322.e9.

29. Kang YK, et al. Humanizing NOD/SCID/ IL-2R $\gamma$ null (NSG) mice using busulfan and retro-orbital injection of umbilical cord blood-derived CD34+ cells. Blood Res. 2016;51(1):31-36.

30. Langley KE, et al. Soluble stem cell factor in human serum. Blood. 1993;81(3):656-660.

31. Choi B, et al. Human B cell development and antibody production in humanized NOD/SCID/ IL-2R $\gamma$ null (NSG) mice conditioned by busulfan. J Clin Immunol. 2011;31(2):253-264.

32. Kim M, et al. Co-transplantation of fetal bone tissue facilitates the development and reconstitution in human B cells in humanized NOD/ SCID/IL-2R $\gamma$ null (NSG) mice. J Clin Immunol. 2011;31(4):699-709.

33. Fineman SM. Optimal treatment of anaphylaxis: antihistamines versus epinephrine. Postgrad Med 2014;126(4):73-81.

34. Honigberg LA, et al. The Bruton tyrosine kinase inhibitor PCI-32765 blocks B-cell activation and is efficacious in models of autoimmune disease and B-cell malignancy. Proc Natl Acad Sci U S A. 2010;107(29):13075-13080.

35. Barf T, et al. Acalabrutinib (ACP-196): a covalent Bruton tyrosine kinase inhibitor with a differentiated selectivity and in vivo potency profile. J Pharmacol Exp Ther. 2017;363(2):240-252.

36. Wu J, Zhang M, Liu D. Bruton tyrosine kinase inhibitor ONO/GS-4059: from bench to bedside. Oncotarget. 2017;8(4):7201-7207.

37. Nam ST, et al. Suppression of IgE-mediated mast cell activation and mouse anaphylaxis via inhibition of Syk activation by 8-formyl-7hydroxy-4-methylcoumarin, $4 \mu 8 \mathrm{C}$. Toxicol Appl Pharmacol. 2017;332:25-31.

38. Li X, Kwon O, Kim DY, Taketomi Y, Murakami M, Chang HW. NecroX-5 suppresses IgE/Ag-stimulated anaphylaxis and mast cell activation by regulating the SHP-1-Syk signaling module. Allergy. 2016;71(2):198-209.

39. Park YH, et al. WZ3146 inhibits mast cell Lyn and Fyn to reduce IgE-mediated allergic responses in vitro and in vivo. Toxicol Appl Pharmacol. 2019;383:114763.

40. Qian F, et al. Scrodentoid A inhibits mast cellmediated allergic response by blocking the LynFceRI $\beta$ interaction. Front Immunol. 2019;10:1103.

41. Deng Y, et al. Sauchinone suppresses FceRImediated mast cell signaling and anaphylaxis through regulation of LKB1/AMPK axis and
SHP-1-Syk signaling module. Int Immunopharmacol. 2019;74:105702.

42. Park YH, et al. Repositioning of anti-cancer drug candidate, AZD7762, to an anti-allergic drug suppressing IgE-mediated mast cells and allergic responses via the inhibition of Lyn and Fyn. Biochem Pharmacol. 2018;154:270-277.

43. Kato T, et al. JTE-852, a novel spleen tyrosine kinase inhibitor, blocks immunoglobulin Gmediated cellular responses and autoimmune reactions in vivo. Life Sci. 2017;191:166-174.

44. Burton OT, et al. Immunoglobulin E signal inhibition during allergen ingestion leads to reversal of established food allergy and induction of regulatory T cells. Immunity. 2014;41(1):141-151.

45. Weber ANR, Bittner Z, Liu X, Dang TM, Radsak MP, Brunner C. Bruton's tyrosine kinase: an emerging key player in innate immunity. Front Immunol. 2017;8:1454.

46. Shillitoe B, Gennery A. X-linked agammaglobulinaemia: outcomes in the modern era. Clin Immunol. 2017;183:54-62.

47. Salem JE, et al. Cardiovascular toxicities associated with ibrutinib. JAm Coll Cardiol. 2019;74(13):1667-1678.

48. Dispenza MC, Regan JA, Bochner BS. Potential applications of Bruton's tyrosine kinase inhibitors for the prevention of allergic reactions. Expert Rev Clin Immunol. 2017;13(10):921-923.

49. Byrd JC, et al. Acalabrutinib (ACP-196) in relapsed chronic lymphocytic leukemia. $\mathrm{NEnglJ}$ Med. 2016;374(4):323-332.

50. Sun CCL, et al. Clinical and biological implications of target occupancy in CLL treated with the BTK inhibitor acalabrutinib [published online March 20, 2020]. Blood. https://doi.org/10.1182/ blood.2019003715.

51. Blank U, Rivera J. Assays for regulated exocytosis of mast cell granules. Curr Protoc Cell Biol. 2006; Chapter 15:Unit 15.11.

52. Adkinson NF. Measurement of total serum immunoglobulin $\mathrm{E}$ and allergen-specific immuno globulin E antibody. In: Rose NR, Friedman H, eds. Manual of Clinical Immunology. American Society of Microbiology; 1980:590-602.

53. Wei Y, et al. Mast cell-specific expression of human Siglec-8 in conditional knock-in mice. Int J Mol Sci. 2018;20(1):E19.

54. Cheng LE, Sullivan BM, Retana LE, Allen CD, Liang HE, Locksley RM. IgE-activated basophils regulate eosinophil tissue entry by modulating endothelial function. J Exp Med. 2015;212(4):513-524.

55. Dwyer DF, Barrett NA, Austen KF, Immunological Genome Project Consortium. Expression profiling of constitutive mast cells reveals a unique identity within the immune system. Nat Immunol. 2016;17(7):878-887.

56. Li XM, Schofield BH, Huang CK, Kleiner GI, Sampson HA. A murine model of IgE-mediated cow's milk hypersensitivity. J Allergy Clin Immunol. 1999;103(2 pt 1):206-214. 